\title{
EGFR exon 19 deletion is associated with favorable overall survival after first-line icotinib therapy in advanced non-small cell lung cancer patients: a retrospective, multiple-center, real- world study
}

\author{
Chuantao Zhang ${ }^{1,2, *}$, Helei Hou ${ }^{1,2, *}$, Xin Cheng ${ }^{3}$, Dianxia Gong ${ }^{4}$, Ling Yang ${ }^{5}$, Haixin \\ Liu $^{5}$, Wenyong Zhu ${ }^{6}$, Na Zhou ${ }^{1,2}$, Yan Zhu ${ }^{5}$, Ling Chen ${ }^{5}$, Kewei Liu ${ }^{1,2}$, Jingjuan Zhu ${ }^{1,2}$,

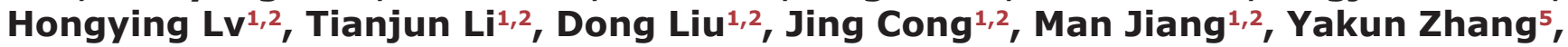 \\ Hualong $\mathbf{Y u}^{1,2}$, Hongsheng $\mathrm{Yu}^{1,2}$ and Xiaochun Zhang ${ }^{1,2}$ \\ ${ }^{1}$ Qingdao University, Qingdao, Shandong, China \\ ${ }^{2}$ Department of Medical Oncology, The Affiliated Hospital of Qingdao University, Qingdao, Shandong, China \\ ${ }^{3}$ Department of Medical Oncology, Qingdao Center Medical Group, No. 2 Affiliated Hospital of Qingdao University, Qingdao, \\ Shandong, China \\ ${ }^{4}$ Department of Medical Oncology, Ju Xian People's Hospital, Rizhao, Shandong, China \\ ${ }^{5}$ Department of Medical Oncology, Qingdao Municipal Hospital, No. 3 Affiliated Hospital of Qingdao University, Qingdao, \\ Shandong, China \\ ${ }^{6}$ Department of Thoracic Surgery, Qilu Hospital of Shandong University, Qingdao, Shandong, China \\ "These authors contributed equally to this work \\ Correspondence to: Xiaochun Zhang, email: zhangxiaochun9670@126.com
}

Keywords: non-small cell lung cancer; epidermal growth factor receptor mutation; icotinib

Received: September 04, $2017 \quad$ Accepted: December 15, $2017 \quad$ Published: December 26, 2017

Copyright: Zhang et al. This is an open-access article distributed under the terms of the Creative Commons Attribution License 3.0 (CC BY 3.0), which permits unrestricted use, distribution, and reproduction in any medium, provided the original author and source are credited.

\section{ABSTRACT}

Background: Although previous studies demonstrated a PFS prolonging trend in NSCLC EGFR exon 19 deletion subgroup comparing with exon 21 L858R mutation subgroup treated with geftinib, erlotinib or afatinib, the efficacy of icotinib as the first-line therapy with sensitive EGFR mutation has not been elucidated.

Patients and Methods: From Sept 20, 2012 to Apr 26, 2016, patients with IIIB/IV NSCLC and a confirmed EGFR exon 19 deletion or exon 21 L858R mutation receiving oral icotinib as first-line therapy were analyzed retrospectively in four cancer centers.

Results: In total, 1615 Chinese patients with advanced NSCLC were screened, and 79 patients receiving icotinib in a first-line setting with intact follow-up data were enrolled. The objective response rate (ORR) was $45.57 \%(36 / 79)$, and the disease contral rate(DCR) reached $89.87 \%(71 / 79)$. The median progress free survival(PFS) and overall survival (OS) for the overall population were 13.61 months and 31.11 months respectively. The median PFS was similar between the EGFR exon 19 deletion subgroup and the exon 21 mutation subgroup (12.30 months vs 14.0 months, $p=$ 0.441, HR 0.90). But the exon 19 deletion group had a significantly longer median OS than the exon 21 mutation group (34.72 months vs 28.66 months, $p=0.036, \mathrm{HR}$ $0.50)$. Meanwhile, there was a significant difference in overall survival in the different ECOG PS subgroups (ECOG PS 0-1 vs ECOG PS 2-3, 32.59 months vs 20.59 months, $p=0.002$, HR 3.09).

Conclusions: EGFR exon 19 deletion is associated with favorable overall survival after first-line icotinib in advanced non small cell lung cancer patients. 


\section{INTRODUCTION}

Non-small cell lung cancer (NSCLC) is the most frequently diagnosed cancer and is a major cause of cancer-related death globally. In 2012, an estimated 1.8 million new lung cancer cases occurred, accounting for approximately $13 \%$ of total cancer diagnoses [1]. Approximately half of newly diagnosed NSCLC patients have advanced disease, and EGFR-TKI (i.e., gefitinib, afatinib and erlotinib) or platinum doublet chemotherapy is the standard therapy choice as the first line for patients according to different driver genes.

Icotinib is an orally administered EGFR tyro-sine kinase inhibitor made in China. The phase 3 study ICOGEN [2] demonstrated that in patients who did not respond to one or more platinum doublet therapy, icotinib was non-inferior to gefitinib in terms of progression-free survival (HR 0.84, 95\% CI 0.67-1.05; median progression-free survival 4.6 months [95\% CI 3.5-6.3] vs 3.4 months [2.3-3.8]; $p=0.13$ ). Approximately $90 \%$ of EGFR mutations are either short in-frame deletions in exon 19 or point mutations that result in a substitution of arginine for leucine at amino acid 858 (L858R) [3]. Several trials confirmed that the response rate to gefitinib, erlotinib or afatinib in patients with EGFR mutations is approximately $62-80 \%$.

According to analyses of American and European clinical trials that assessed first-line TKI treatment, patients with exon 19 deletions have a longer median TTP (14.6 versus 9.7 months; $p=0.02)$ and OS (30.838.0 versus $14.8-17.0$ months; $p<0.05$ ) compared with those harboring the exon 21 L858R mutation. Although a prolonging trend in PFS or OS was identified in the exon 19 deletion subgroup compared with the L858R subgroup in advanced NSCLC patients in the IPASS [4], NEJ002 [5], WJTOG3405 [6], and LUX LUNG3 [7] studies, no statistical analysis was performed to clarify the differences among these groups. Therefore, whether there is a difference in PFS and OS among these groups remains controversial. Meanwhile, no prospective study on the efficacy of NSCLC patients treated with icotinib as the first-line therapy has been published, and whether icotinib can prolong the PFS and OS between exon 19 deletion and exon 21 L858R mutation is unknown. To address this issue, we performed this retrospective study to test the hypothesis that advanced NSCLC patients with an EGFR exon 19 deletion who are treated with icotinib as the first-line treatment will have prolonged PFS and OS compared to patients with an exon $21 \mathrm{~L} 858 \mathrm{R}$ mutation.

\section{MATERIALS AND METHODS}

\section{Study design and treatment}

This study is a retrospective analysis of all consecutive stage IIIB/IV NSCLC Chinese patients with an EGFR exon 19 deletion or exon 21 L858R mutation treated with icotinib as the first-line therapy from Sept 20, 2012 to Apr 26, 2016. A total of 4 cancer centers in Qingdao participated in this study.

\section{Drug administration}

Patients received $125 \mathrm{mg}$ oral icotinib in tablets three times per day until progression of disease, occurrence of intolerable severe toxicity, or death.

For patients who had grade 3 or 4 adverse events, dose reductions of icotinib were applied, but treatment was allowed to be interrupted for up to 14 days.Local therapy was allowed to be given (eg. palliative surgery, SRT, WBRT, intrapleural chemotherapy, $\mathrm{I}^{125}$ ablation, etc.) with icotinib.

\section{Patients}

The inclusion criteria were as follows: 1) patients older than 18 years with histologically or cytologically confirmed advanced or recurrent stage IIIB or IV NSCLC (Union for International Cancer Control classification 7th edition [8]) and a confirmed activating mutation of EGFR (an exon 19 deletion or an exon 21 L858R mutation); 2) chemotherapy or EGFR-TKI naive (although adjuvant or neo-adjuvant therapy was allowed for non-metastatic disease in which relapse had occurred $\geq 6$ months after final treatment); 3) measurable disease according to Response Evaluation Criteria In Solid Tumors (RECIST version 1.1 [9]) and Eastern Cooperative Oncology Group [10] performance status of $0-3 ; 4$ ) adequate hematological, biochemical, and organ function.

Meanwhile, symptomatic brain metastasis and local palliative therapy (i.e. radiotherapy, $\mathrm{I}^{125}$ ablation, and surgery) were allowed.All patients received at least one dose of the study drug.

The exclusion criteria were: 1) patients diagnosed at one hospital but thereafter transferred to other hospitals for aggressive treatment; 2) patients who received diagnosis and introduction of any aggressive treatment not performed at the four centers and who were thereafter transferred to one of the four centers for later stage treatment; 3) patients with impaired cardiac function, severe liver or renal disease, inadequate bone marrow reserve, or uncontrolled diabetes; 4) patients in whom EGFR mutations were not examined or the results were not available; 5) untreated patients and patients with missing survival details; and 6) pregnant or lactating patients or patients whose participation in the trial was judged to be inappropriate by the attending doctor.

Data were collected from the time of primary NSCLC diagnosis until death or the end of the study period. The baseline clinical characteristics included age, sex, ECOG PS, physical staged diagnosis, tumor histology, smoking history, EGFR mutation point, etc. 
This multicentric study was approved by the institutional review board of each participating institute. All of the patients provided written informed consent before participation in the study.

\section{Progression-free survival, overall survival and tumor response}

The primary endpoint was overall survival (OS), which was defined as the time from the date of beginning treatment to the date of death or the last follow-up. The second endpoint included progression-free survival (PFS), objective response rate $(\mathrm{ORR}=\mathrm{CR}+\mathrm{PR})$, and disease control rate $(\mathrm{DCR}=\mathrm{CR}+\mathrm{PR}+\mathrm{SD})$. $\mathrm{PFS}$ was defined as the time from the beginning of treatment to the earliest sign of disease progression, as determined by CT, ultrasound or MRI imaging using RECIST 1.1 criteria, or death from any cause. The assessment of efficacy was done by the designated physicians, who worked for over 20 years and had senior professional title in lung cancer research. And if there was some difficulty to define, Hualong Yu, a imaging specialist who has Medical degree and studied lung cancer radiology tomography for 18 years, helped to evaluate and define the results.

\section{Follow-up}

Follow-up was performed first-month after beginning treatment, two to three months in two years, and then in six-month intervals until a five-year follow-up period. The follow-up included medical history, clinical examination, chest $\mathrm{CT}$ in all patients. In case of clinical or radiological suspicion of local recurrence or metastatic disease, specific imaging evaluations (i.e. CT, ECT or MRI scan) were performed. The interval of assessment was at the physician's discretion. The patients were followed up until death or lost visit.

\section{Statistical analyses}

Continuous, discrete, and categorical variables are expressed as the mean \pm standard deviation, median (range), and frequency, respectively. The normality of distribution and homogeneity of variances were assessed by Shapiro-Wilk and $F$-tests, respectively. To compare relative frequencies, discrete variables, and normally distributed continuous variables between genotype subgroup, we used $\chi^{2}$ or Fisher's exact test. Logistic regression analysis was used to find factors influencing outcome variables and survival after icotinib therapy. We arbitrarily excluded either of the coefficients from candidate explanatory variables in the multivariate analysis. These results were expressed as hazard ratios (HRs) and 95\% confidence intervals (CIs). A p-value 0.05 was statistically significant, and all $p$ values were twosided. The survival time of patients who were known to be alive at the time of the data update were censored at the date of last follow-up. Median follow-up time, progression-free survival and overall survival were estimated by the Kaplan-Meier method, and the log-rank test was used to compare the difference between genotype groups. The hazard ratio (HR) between two groups was estimated by proportional hazards regression with a 95\% Wald confidence interval (95\% CI). Data analysis was performed with SPSS software (version 23.0; IBM Corporation, Armonk, NY) for all statistical analyses.

\section{RESULTS}

\section{Patient characteristics at baseline}

Between Sept 20, 2012 and Apr 26, 2016, a total of 1615 NSCLC lung cancer patients received icotinib. Of them, $712(40.08 \%)$ patients had intact follow-up data, and 81 (11.38\%) had sensitive EGFR mutations and received icotinib as the first-line therapy. Seventy-nine of $712(11.10 \%)$ patients had an EGFR exon 19 deletion or L858R substitution in exon 21 (27 patients with an exon 19 deletion, 52 patients with an L858R in exon 21) (Figure 1).

The median age at the time of enrollment was 64.17 years (range, $41-83$ years). Of the 79 patients enrolled, $51(64.56 \%)$ were women and $28(35.44 \%)$ were men. The number of patients with ECOG PS 0, 1, 2, and 3 was 7 (9.88\%), 50 (63.29\%), $16(20.25 \%)$ and 6 (7.59\%), respectively. All of the patients had adenocarcinoma $(76,96.20 \%)$ except for one squamous carcinoma (1, $1.27 \%)$, one sarcomatoid carcinoma $(1,1.27 \%)$ and one adenosquamous carcinoma $(1,1.27 \%)$. There were 8 (10.11\%) patients with stage IIIB, $69(87.34 \%)$ patients with stage IV, and $2(2.53 \%)$ patients with postoperative recurrence. Twenty-seven $(34.18 \%)$ patients had EGFR exon 19 deletion mutations, and $52(65.82 \%)$ patients had L858R substitution mutations in exon 21. Both treatment groups were generally well matched with respect to baseline characteristics (Table 1). Baseline characteristics are described in Table 1. The median follow-up was 24.46 months (range 1.0-72 .0 months). At the data cutoff point (Apr 28, 2017), 54 (68.35\%) patients had PD, 36 patients $(45.57 \%)$ were deceased, 5 patients $(6.33 \%)$ failed to be followed-up.

\section{Duration of treatment, response and survival}

The median duration of treatment was 13.0 months (range 1.0-31.90) for icotinib.The objective response rate $(\mathrm{ORR}=\mathrm{CR}+\mathrm{PR})$ was $36(45.56 \%)$. One $(2.2 \%)$ patient had CR, 35 (44.30\%) patients had PR, 35 (44.30\%) patients had $\mathrm{SD}$, and $8(10.13 \%)$ patients had PD. The resulting disease control rate $(\mathrm{DCR}=\mathrm{CR}+\mathrm{PR}+\mathrm{SD})$ reached $89.87 \%$.

The median OS was 34.72 months (95\% CI, 26.4243.02 months) in the EGFR exon 19 deletion subgroup; the 1 -year OS rate was $92.59 \%(25 / 27)$, the 2 -year OS was 
Table 1: Demographic data of all patients $(N=79)$

\begin{tabular}{|c|c|c|c|}
\hline Characteristics & EGFR exon 19 deletion, N. (\%) & EGFR exon 21 L858R substitution N.(\%) & $P$ Value \\
\hline \multicolumn{4}{|l|}{ Age } \\
\hline$\leq 65$ & $18(66)$ & $30(58)$ & 0.365 \\
\hline$>65$ & $9(33)$ & $22(42)$ & \\
\hline \multicolumn{4}{|l|}{ Sex } \\
\hline Male & $8(30)$ & $20(38)$ & 0.088 \\
\hline Female & $19(70)$ & $32(62)$ & \\
\hline \multicolumn{4}{|l|}{ Smoking status } \\
\hline somoker & $6(22)$ & $11(21)$ & 0.918 \\
\hline never-smoking & $21(78)$ & $41(79)$ & \\
\hline \multicolumn{4}{|l|}{ Histology } \\
\hline adenocarcinoma & $25(93)$ & $51(98)$ & \\
\hline Non-adenocarcinoma & $2(7)$ & $1(2)$ & \\
\hline \multicolumn{4}{|l|}{ Disease stage } \\
\hline IIIB & $2(7)$ & $5(10)$ & \\
\hline IV & $24(89)$ & $46(89)$ & \\
\hline recurrence & $1(3.70)$ & $1(1.92)$ & \\
\hline \multicolumn{4}{|l|}{ ECOG PS } \\
\hline $0-1$ & $18(67)$ & $39(75)$ & 0.556 \\
\hline $2-3$ & $9(33)$ & $13(25)$ & \\
\hline \multicolumn{4}{|l|}{ Brain metastasis } \\
\hline Yes & $0(0)$ & $4(8)$ & \\
\hline No & $27(100)$ & $48(92)$ & \\
\hline \multicolumn{4}{|l|}{ Local therapy } \\
\hline Yes & $9(32)$ & $11(22)$ & 0.219 \\
\hline No & $18(68)$ & $41(79)$ & \\
\hline \multicolumn{4}{|l|}{ Subsequent therapy } \\
\hline yes & $7(26)$ & $18(35)$ & 0.431 \\
\hline no & $20(74)$ & $34(65)$ & \\
\hline
\end{tabular}

Data are $n(\%)$ or median (range) and are for all patients with confirmed disease who received at least one dose of study drug.

*Non-adenocarcinoma squamous-cell carcinoma $(n=1)$, adenosquamous carcinoma $(n=1)$, and sarcomatoidcarcinoma $(n=1)$. Abbreviations: $\mathrm{ECOG}=$ Eastern Oncology Group; PS = performance status.

$37.04 \%(10 / 27)$, and the 3-year OS was $14.81 \%(4 / 27)$. The median OS was 28.66 months (95\% CI, 24.83-32.48 months) in the EGFR exon 21 mutation subgroup, the 1-year OS was 42/52 (78.84\%), the 2-year OS was 20/52 $(38.46 \%)$, and the 3 -year OS was $4 / 52$ (7.69\%). There was a significant difference in OS between the EGFR exon 19 deletion subgroup and EGFR exon 21 mutation subgroup $(p=0.036)$.

After univariate analysis of OS, there was no significant difference between male and female patients, smokers and never-smokers, age $\leq 65$ years and $>65$ years. However, there was significant difference between
ECOG PS 0-1 and PS 2-3 (32.59 months vs 28.53 months, $p=0.011)$, age $\leq 65$ years and $>65$ years $(34.72$ months vs 21.41 months, $p=0.021)$, EGFR exon 19 deletion subgroup and exon 21 L858R subgroup (34.72 months vs 21.41 months, $p=0.021)$. After multivariate analysis, there was a difference in OS between the EGFR exon 19 deletion subgroup and EGFR exon 21 mutation subgroup $(\mathrm{HR}=0.31,95 \%$ CI 0.13-0.71, $p=0.006)$ (Figure 2), ECOG PS 0-1 subgroup and PS 2-3 subgroup (HR = 3.36, 95\% CI 1.63-6.97, $p=0.006$ ) (Figure 3).

The median PFS of the overall population was 13.61 months (95\% CI, range 10.70-16.51 months), the 1-year 
PFS was 36/79 (45.60\%), and the 2-year PFS was 10/79 (12.65\%). The median OS was 31.12 months $(95 \% \mathrm{CI}$, range $27.70-35.13$ months), the 1 -year OS was $67 / 79$ (84.81\%), the 2-year OS was $30 / 79$ (37.97\%), and the 3 -year OS was $8 / 79$ (10.13\%). The median PFS was 12.30 months (95\% CI, 5.70-18.89 months) in the EGFR exon 19 deletion subgroup; the 1-year PFS rate was $44.44 \%$ $(12 / 27)$, and the 2 -year PFS was $7.41 \%(2 / 27)$. The median PFS was 13.64 months (95\% CI, 9.78-17.50 months) in the exon 21 mutation subgroup; the 1-year PFS was 24/52 (46.15\%), and the 2-year PFS was 8/52 (15.38\%). There is no difference in PFS between the EGFR exon 19 deletion subgroup and exon 21 mutation subgroup ( $p=0.44$ ) (Figure 4). There was no significant difference in PFS between male and female patients (10.36 months vs 13.60 months, $p=0.652)$, the ECOG PS 0-1 subgroup and PS 2-3 subgroup (12.33 months vs 15.61 months, $p=0.411$ )
(Figure 5 ), age $\leq 65$ years and $>65$ years ( 9.96 months vs 16.74 months, $p=0.101)$, and smokers and non-smokers (12.33 months vs 17.44 months, $p=0.976)$.

\section{Subsequent therapy}

Patient management after the icotinib treatment was also retrospectively investigated. Of the 54 patients who had PD, 27 patients were given subsequent therapy. Of them, 7 patients were given continual icotinib, 13 patients were given chemotherapy containing platinum doublets, 2 patients were given erlotinib or gefitinib, 2 patients were given AZD 9291, and 4 patients were given local therapy, including WBRT, intrapleural chemotherapy, and $\mathrm{I}^{125}$ ablation. There was no significant difference in post-therapy between the exon 19 deletion and exon 21 mutation subgroups $(p=0.431)$.

\section{5 cases with stage IIIB/IV NSCLC patients receiving icotinib}

(From Sept 20.2012 to Apr 26. 2016)

789 cases with intact follow-up data receiving icotinib

221 cases receiving icotinib in second line setting

285 cases receiving icotinib in third line setting

194 cases receiving icotinib in other line setting

85 cases with EGFR sensitive mutation receiving icotinib in first line setting

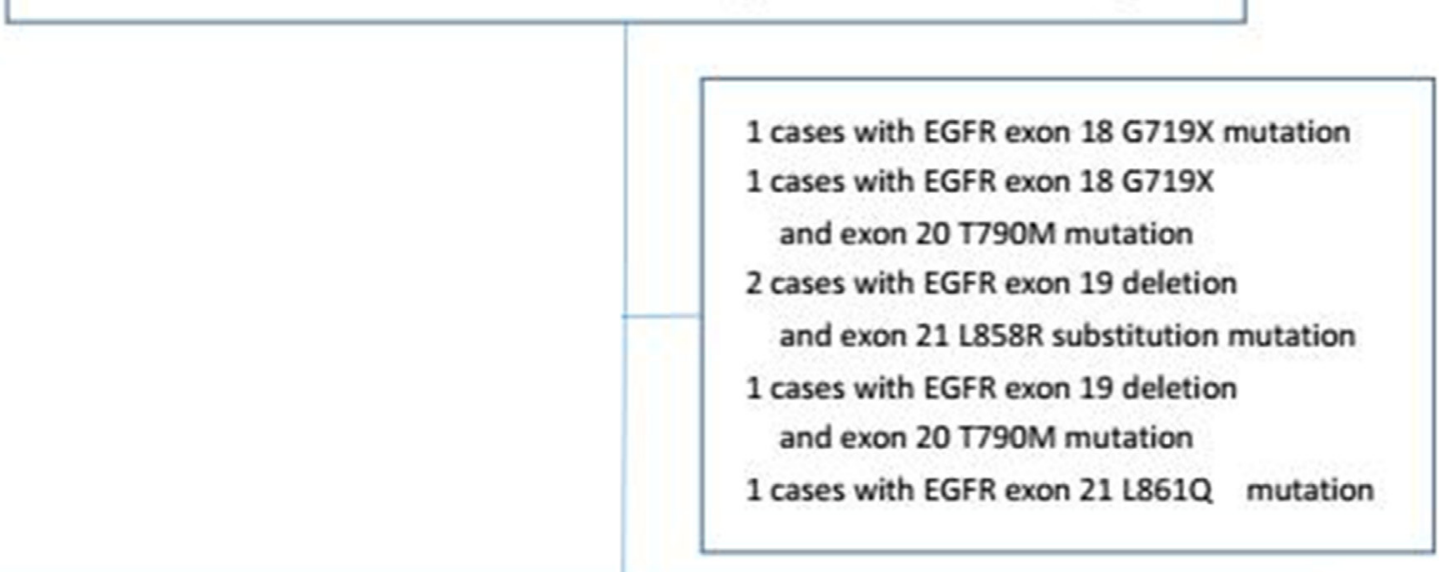

79 cases enrolled with EGFR exon 19 deletion(n=27) or exon 21 L85R substitution mutation(n=52)

27 cases with EGFR exon 19 mutation

52 cases with EGFR exon 19 mutation

Figure 1: The flow chart of this study. 


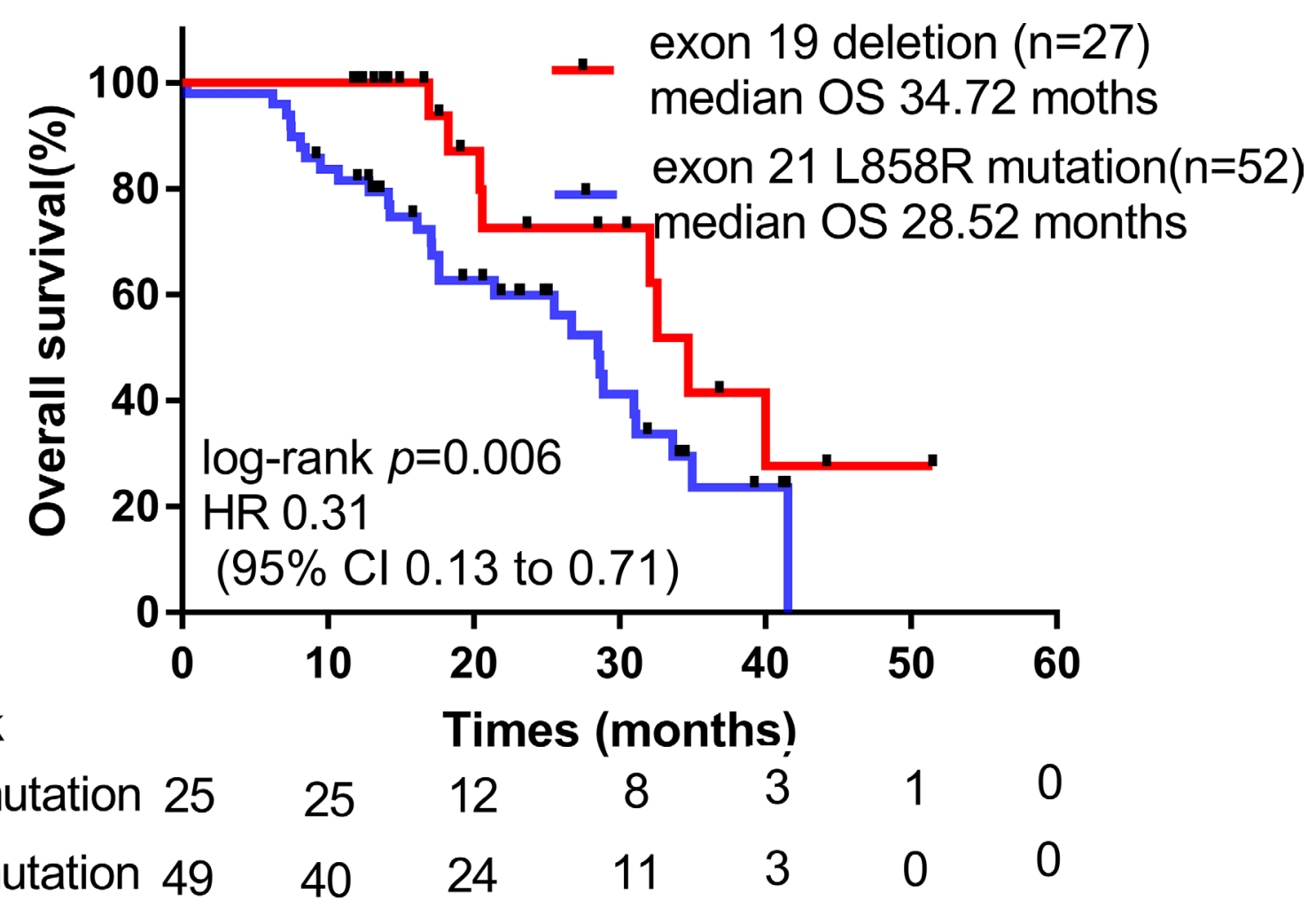

Figure 2: OS analysis between EGFR exon 19 del subgroup and exon 21 L858R substitution subgroup.

No. at risk

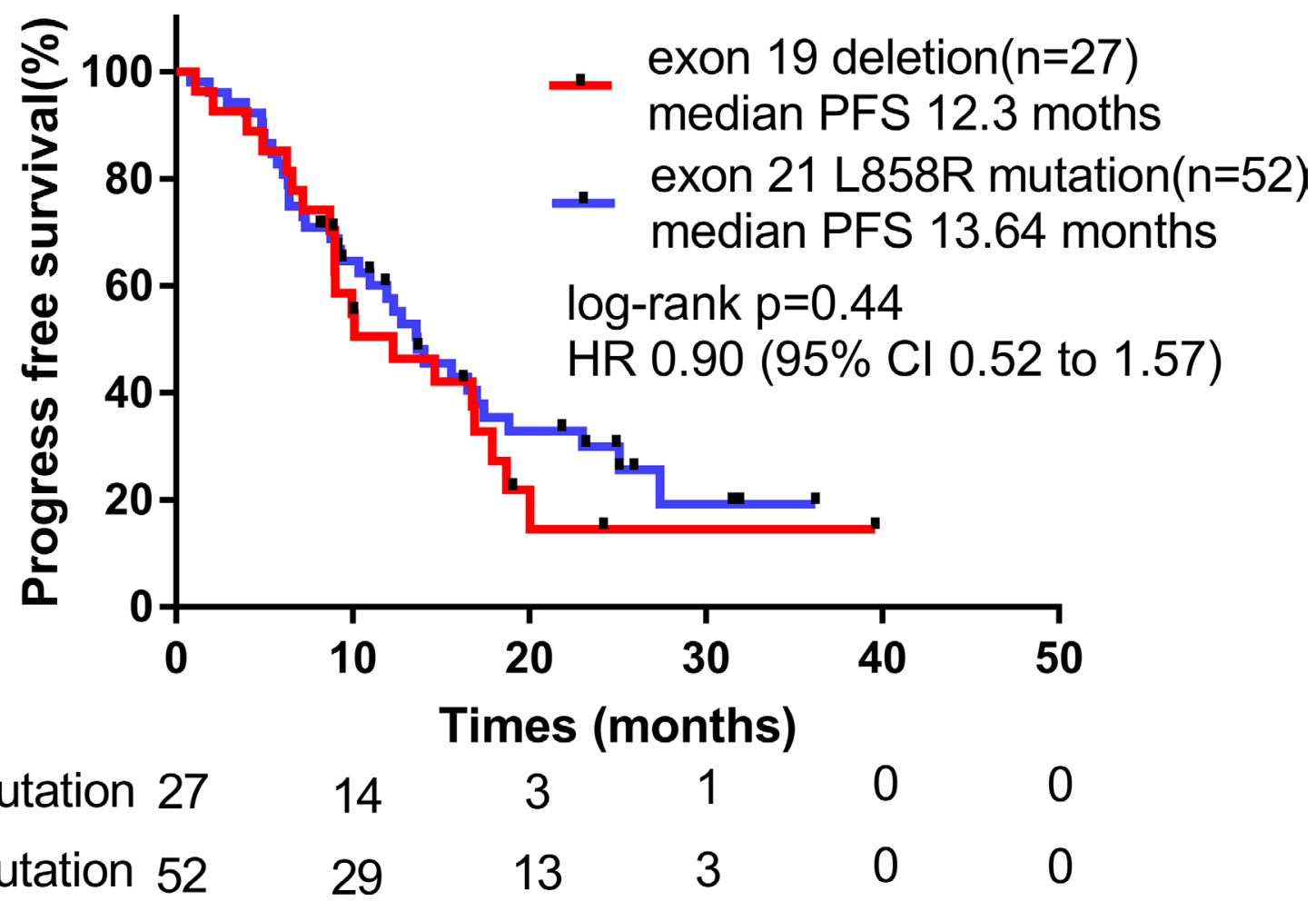

Figure 3: Comparison of PFS between EGFR exon 19 del subgroup and 21 L858R substitution subgroup. 


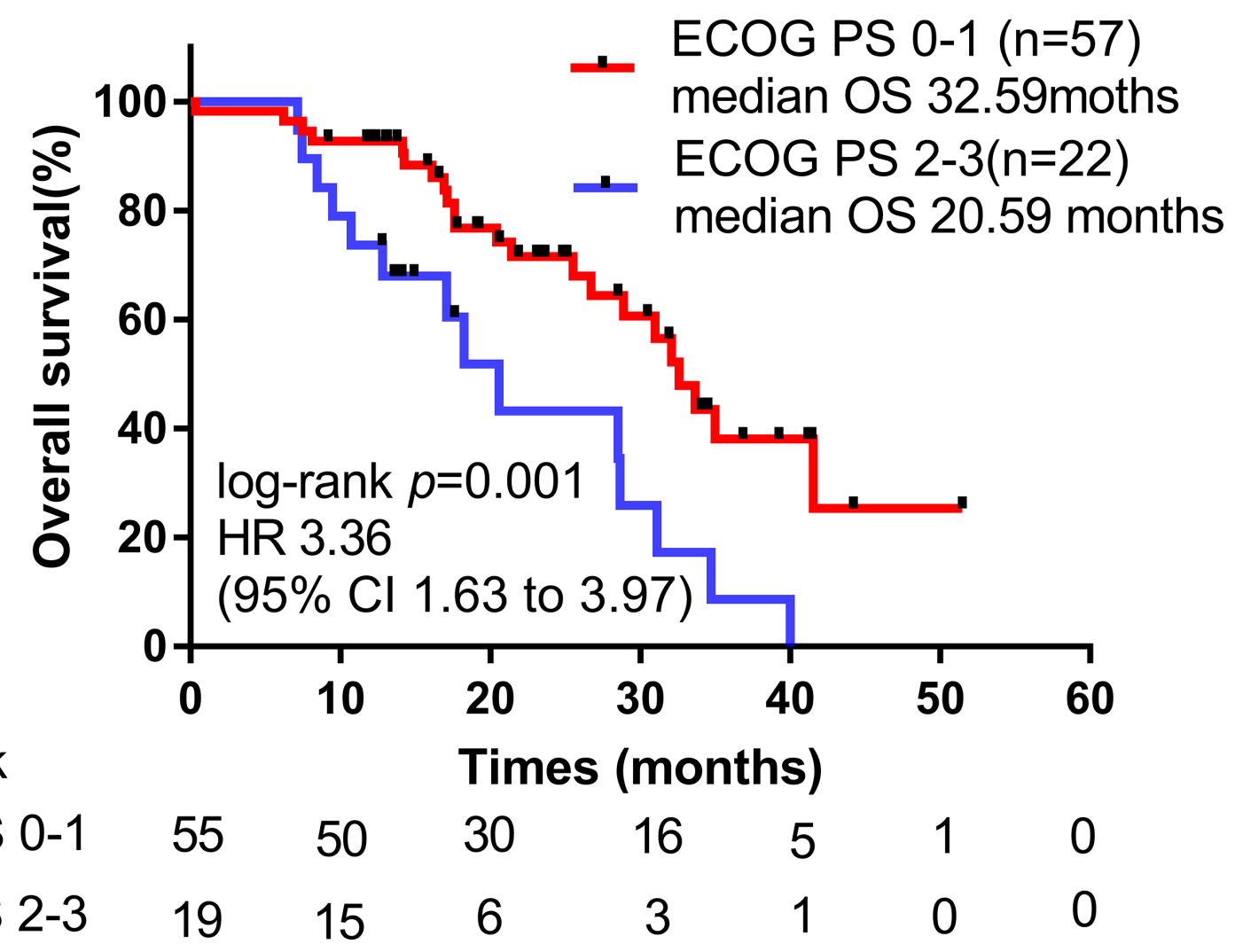

Figure 4: OS analysis based on ECOG PS.

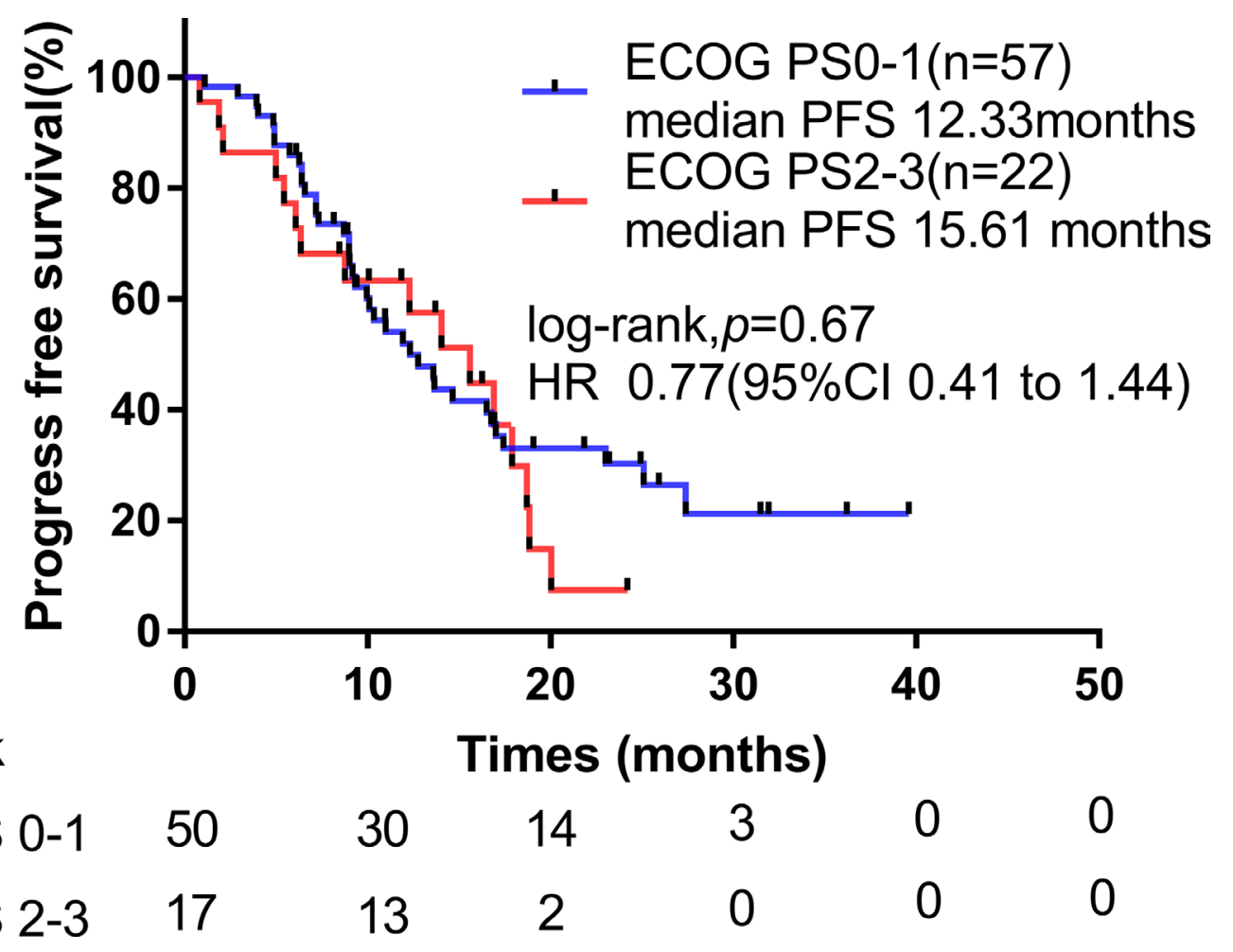

Figure 5: Comparison of PFS between ECOG PS 0-1 subgroup and ECOG PS 2-3 subgroup. 


\section{DISCUSSION}

Icotinib, a novel EGFR-TKI made in China, first exhibited a PFS non-inferior to gefitinib, and icotinib has superior safety and tolerability compared with gefitinib in an RCT phase 3 clinical ICOGEN trial [2] in patients with advanced NSCLC previously treated with chemotherapy. Our study confirmed an encouraging ORR, PFS and OS if the NSCLC patients with EGFR mutation were treated with icotinib as the first-line treatment, which is similar to previous studies on gefitinib and erlotinib in patients with EGFR mutation-positive advanced NSCLC. In our study, the ORR and DCR of icotinib were $45.56 \%$ and $89.87 \%$, respectively, and the median PFS and OS were 13.60 (95\% CI 10.70-16.51) months and 31.11 months, respectively. In the IPASS [4], OPTIMAL [11], WJTOG34O5 [6], NEJ002 [12], and EURAC [13] clinical studies, gefitinib or erlotinib yielded a prolonged median PFS compared with chemotherapy (9.2-13.1 months versus 4.6-6.3 months) and an increased response rate (64\%-82\% versus $30.7-47.3 \%$ ) compared with chemotherapy for patients with sensitive EGFR mutations. Meanwhile, the efficacy of icotinib in this study is similar to previous retrospective studies, which showed an ORR of $48.6 \%-66.7 \%$, a DCR of 94.4-95.6\%, a median PFS of 11.0-14.9 months, and a median OS of 21.0-37.0 months [14, 15].

The median ORR and FPS of icotinib in our study seem higher compared with gefitinib or erlotinib. The reason may be related to the local therapy added to the patients in our study. Compared with gefitinib or erlotinib, the median PFS of icotinib in this study is higher, possibly related to $34.18 \%(27 / 79)$ of patients receiving local therapy (eg. as palliative surgery, radiotherapy, WBRT, SRS, ${ }^{125}$ ablation therapy, etc).

In our study, the median PFS of icotinib was 13.61 months in the overall population, 12.3 months in the EGFR exon 19 deletion subgroups, and 14.0 months in the 21 mutation subgroups, which demonstrates that icotinib is not inferior to gefitinib or erlotinib as a first-line therapy. However, this finding should be further explored in a prospective study. In fact,the CONVENCE study [16] of first-line icotinib versus cisplatin/pemetrexed plus pemetrexed maintenance therapy in lung adenocarcinoma patients with EGFR mutations showed the median PFS of the icotinib group was 9.9 months, and it was 8.9 and 8.3 months in the EGFR exon 19 and exon 21 subgroups, respectively ( $p$ $=0.3605$ ). But the OS of this study has not been reported yet.

Previous RCT research including the IPASS, OPTIMAL didn't compare the PFS or OS in patients harboring EGFR exon 19 del with those harboring exon 21 L858R mutation, but a meta-analysis [17] revealed significant OS benefits in patients harboring exon $19 \mathrm{del}$ (HR: $0.72,95 \%$ CI: 0.60-0.88) comparing with L858R (HR: 1.15, 95\% CI: 0.95-1.39) . Meanwhile, according to the analyses of American and European clinical trials [18] that assessed first-line TKI treatment, patients with an exon 19 deletion had a significantly longer time to progress (TTP) (14.6 versus 9.7 months; $p=0.02$ ) and OS than those with exon 21 L858R (30.8 vs 14.8 months; $p<0.0001)$. Additionally, in Spanish, Kerean and Chinese studies, there were similar results [19-21]. In our study, the patients in the EGFR exon 19 deletion subgroup had a longer OS than the patients in the EGFR exon 21 mutation subgroup (34.72 months versus 28.66 months). In our opinion, first-generation EGFR TKI, including icotinib as a first-line therapy, can yield a longer OS in patients with an EGFR exon 19 deletion than those with an exon 21 L858R mutation.

However, in our study, there was no difference in PFS between EGFR exon 19 del subgroup and exon 21 L858R subgroup. The other mechanism may be related to icotinib, which may be different from gefitnib, erlotinib and afatinib. In fact, in the CONVIENCE [1] study, there was not PFS difference seen between EGFR exon 19 and exon 21 subgroups either.

In a subgroup analysis, no significant difference in PFS was observed between subgroups of sex, age, smoking history, ECOG PS, and EGFR mutation type subgroups. Nevertheless, significant differences in OS were observed during different ECOG PS, age and EGFR mutation type subgroups, but no difference in OS was observed for subgroups of sex, smoking history, or stage of disease .

Meanwhile, the subgroups of age $>65$ years, ECOG PS 2-3 and L858R mutation in EGFR have poor prognoses. The results are consistent with a study comparing EGFR exon 19 with L858R subgroups of 166 NSCLC patients with different EGFR mutations treated with second-line TKIs [19, 20], which revealed that the exon 19 deletion group had a significantly longer OS (13.7 vs 11.7 months, $p$ $=0.02$ ) compared with the exon 21 L858R mutation group. A meta-analysis of 13 eligible trials involving 912 patients [21] showed that the two-year survival rate of the 19 deletion group was significantly higher than the $21 \mathrm{~L} 858 \mathrm{R}$ group (OR 5.27, 95\% CI 1.76-15.71; $p=0.003$ ). There were similar results in a meta-analysis of icotinib [22, 23]. Therefore, EGFR exon 19 deletion is associated with favorable OS after first-line gefitinib therapy in advanced non-small cell lung cancer patients.

Although the mechanism is uncertain, some research articles showed the first generation EGFR-TKI could induce T790M mutation to occur in the NSCLC patients with resistance $[24,25]$. In fact, there is low T790M mutation incidence $(2.7 \%)$ in pretreated NSCLC patients, but high T790M mutation incidence (36\%) in the NSCLC patients with EGFR-TKI resistance [24, 25]. Another study showed OS benefit in patients with T790M mutation [26]. Therefore, we can say EGFR-TKI can induce T790M mutation. Meanwhile there is a larger proportion of T790M mutation in NSCLC patients harbouring exon 19 del than those harbouring exon 21 L858R mutation, that may be the mechanism of EGFR-TKI contribute to the better survival of patients with exon 19 del [26]. And so, 
our conclusion of manuscript is rationale partially.

Additionally, this study showed that the patients with EGOC PS 0-1 and EGFR exon 19 del had longer OS, suggesting the clinical background of patients affects clinical efficacy. This knowledge should lead to molecularly based and more personalized treatment of advanced NSCLC.

In a phase 4 clinical trial [27] containing 6,087 patients, icotinib demonstrated a favorable toxicity profile in the routine clinical setting, so this study does not refer to safety.

\section{CONCLUSIONS}

The results of this study illustrate that icotinib is effective as the first-line treatment for patients with advanced NSCLC harbouring sensitive EGFR mutations. The patients harbouring EGFR exon 19 del had the similar median PFS, but longer OS comparing with those harbouring exon 21 L858R mutation. Icotinib may be the optimal choice in patients harbouring EGFR exon 19 del.

\section{Abbreviations}

PFS: progression-Free Survival; OS: overall Survival; ORR: objective response rate; DCR: disease control rate; NSCLC: non small cell lung cancer; CR: complete response; PR: partial response; SD: stable disease; PD: progression of disease; EGFR: epidermal growth factor receptor; TKI: tyrosine kinase inhibitor; ECOG PS: Eastern Cooperative Oncology Group Performance Status; TTP: time to progress.

\section{Author contributions}

Xiaochun Zhang was responsible for the conception, design, and financial support of the study. Chuantao ZhangHelei Hou and Hongsheng Yu was responsible for the collection, interpretation of data ,and drafting the article or revising it critically for important intellectual content. Xin Cheng, Dianxia Gong, Ling Yang, Haixin Liu, Wenyong Zhu, Tianjun Li, Hongying Lv, Zhu Jingjuan, Kewei Liu, Yakun Zhang, Yan Zhu and Ling Chen were involved in the collection of clinical data. Dong Liu, Na Zhou, Jing Cong, Man Jiang are take part in the genetic tests research report interpretation. All of the authors participated in writing and reviewing the article and accept full responsibility for its overall content.The study was designed and performed independently of any pharmaceutical company. The report was written by the corresponding author, who had unrestricted access to the study data and is responsible for the accuracy and completeness of the reported analyses. The corresponding author had the final responsibility for the decision to submit for publication.

\section{ACKNOWLEDGMENTS}

The authors would like to thank all participating physicians and patients enrolled. The authors thanked the patients and their family for for their contribution to this articles. No writing assistance was involved in this article.

\section{CONFLICTS OF INTEREST}

No authors received a grant or fees for participation in the review activities for his institution from Beta. All authors declare that they have no conflicts of interest.

\section{FUNDING}

This research was supported by Taishan Scholar foundation [grant numbers: tshw201502061], Qingdao People's Livelihood Science and Technology Program [grant numbers: 16-6-2-3-nsh] and the second batch of Qingdao soft science project [grant numbers: 16-9-2-1-(1)-CHX], which were all from the Chinese Government.

\section{REFERENCES}

1. Siegel R, Naishadham D, Jemal A. Cancer statistics, 2012. CA Cancer J Clin. 2012; 62:10-29.

2. Shi Y, Zhang L, Liu X, Zhou C, Zhang L, Zhang S, Wang D, Li Q, Qin S, Hu C, Zhang Y, Chen J, Cheng Y, et al. Icotinib versus gefitinib in previously treated advanced nonsmall-cell lung cancer (ICOGEN): a randomised, doubleblind phase 3 non-inferiority trial. Lancet Oncol. 2013; 14:953-61.

3. Yun CH, Boggon TJ, Li Y, Woo MS, Greulich H, Meyerson M, Eck MJ. Structures of Lung Cancer-Derived EGFR Mutants and Inhibitor Complexes: Mechanism of Activation and Insights into Differential Inhibitor Sensitivity. Cancer Cell. 2007; 11:217.

4. Fukuoka M, Wu YL, Thongprasert S, Sunpaweravong P, Leong SS, Sriuranpong V, Chao TY, Nakagawa K, Chu DT, Saijo N, Duffield EL, Rukazenkov Y, Speake G, et al. Biomarker Analyses and Final Overall Survival Results From a Phase III, Randomized, Open-Label, First-Line Study of Gefitinib Versus Carboplatin/Paclitaxel in Clinically Selected Patients With Advanced Non-Small-Cell Lung Cancer in Asia (IPASS). J Clin Oncol. 2011; 29:2866-74.

5. Inoue A, Kobayashi K, Maemondo M. Final overall survival results of NEJ002, a phase III trial comparing gefitinib to carboplatin (CBDCA) plus paclitaxel (TXL) as the first-line treatment for advanced non-small cell lung cancer (NSCLC) with EGFR mutations. J Clin Oncol. 2011; 29.

6. Mitsudomi T, Morita S, Yatabe Y, Negoro S, Okamoto I, Tsurutani J, Seto T, Satouchi M, Tada H, Hirashima T, Asami K, Katakami N, Takada M, et al. Gefitinib versus cisplatin plus docetaxel in patients with non-small-cell lung cancer harbouring mutations of the epidermal growth factor receptor (WJTOG3405): an open label, randomised phase 3 trial. Lancet Oncol. 2010; 11:121-8.

7. Yang JC, Sequist LV, Geater SL, Tsai CM, Mok TS, Schuler M, Yamamoto N, Yu CJ, Ou SH, Zhou C, Massey D, Zazulina 
$\mathrm{V}, \mathrm{Wu}$ YL. Clinical activity of afatinib in patients with advanced non-small-cell lung cancer harbouring uncommon EGFR mutations: a combined post-hoc analysis of LUX-Lung 2, LUX-Lung 3, and LUX-Lung 6. Lancet Oncol. 2015; 16:830.

8. Marshall HM, Leong SC, Bowman RV, Yang IA, Fong KM. The science behind the 7th edition Tumour, Node, Metastasis staging system for lung cancer. Respirology. 2012; 17:247-60.

9. Eisenhauer EA, Therasse P, Bogaerts J, Schwartz LH, Sargent D, Ford R, Dancey J, Arbuck S, Gwyther S, Mooney M, Rubinstein L, Shankar L, Dodd L, et al. New response evaluation criteria in solid TUMORs: revised RECIST guideline (version 1.1). Eur J Cancer. 2009; 45:228-47.

10. Oken MM, Creech RH, Tormey DC, Horton J, Davis TE, McFadden ET, Carbone PP. Toxicity and response criteria of the Eastern Cooperative Oncology Group. Am J Clin Oncol. 1982; 5:649.

11. Zhou C, Wu YL, Chen G, Feng J, Liu XQ, Wang C, Zhang S, Wang J, Zhou S, Ren S, Lu S, Zhang L, Hu C. Erlotinib versus chemotherapy as first-line treatment for patients with advanced EGFR mutation-positive non-small-cell lung cancer (OPTIMAL, CTONG-0802): a multicentre, open-label, randomised, phase 3 study. Lancet Oncol. 2011; 12:735-42.

12. Miyauchi E, Inoue A, Kobayashi K, Maemondo M, Sugawara S, Oizumi S, Isobe H, Gemma A, Saijo Y, Yoshizawa H, Hagiwara K, Nukiwa T. First line gefetinib vs carbopaltin/paclitaxel in non small cell lung cancer with EGFR mutation:phase III study NEJ002. Meeting of the Japanese-Society-Of-Medical-Oncology. 2010; 11.

13. Benlloch S, Taron M, Botero ML, Bertran-Alamillo J, Mayo C, Capitan AG, de Aguirre I, Queralt C, Ramirez JL, Rosell R, Klughammer B, Schlegel M, Chen D, et al. Retrospective EGFR mutation testing of clinical specimens from the EURTAC trial of erlotinib in non-small cell lung cancer (NSCLC) using a novel allele-specific PCR (ASPCR) assay. J Clin Oncol. 2012; 30

14. Chen H, Wang HP, Zhang L, Si XY. [The therapeutic value and safety of icotinib as first-line therapy for advanced non-small cell lung cancer patients]. [Article in Chinese]. Zhonghua Nei Ke Za Zhi. 2017; 56:39-43.

15. Nong J, Qin N, Wang J, Yang X, Zhang H, Wu Y, Lv J, Zhang Q, Zhang S. [Clinical effects for patients with recurrent advanced non-small cell lung cancer treated with icotinib hydrochloride]. [Article in Chinese; Abstract available in Chinese from the publisher]. Zhongguo Fei Ai Za Zhi. 2013; 16:240-5.

16. Shi YK, Wang L, Han BH, Li W, Yu P, Liu YP, Ding CM, Song X, Ma ZY, Ren XL, Feng JF, Zhang HL, Chen GY, et al. First-line icotinib versus cisplatine/pemetrexed plus pemetrexed maintenance therapy in lung adenocarcinoma patients with sensitizing EGFR mutation (CONVINCE). Ann Oncol. 2017; 28:2443-2450

17. Kuan FC, Kuo LT, Chen MC, Yang CT, Shi CS, Teng D, Lee KD. Overall survival benefits of first-line EGFR tyrosine kinase inhibitors in EGFR-mutated non-small-cell lung cancers: a systematic review and meta-analysis. Brit J Cancer. 2015; 113:1519-28.

18. Jackman DM, Miller VA, Cioffredi LA. Impact of epidermal growth factor receptor and KRAS mutations on clinical outcomes in previously untreated non-small cell lung cancer patients: results of an online tumor registry of clinical trials. Clin Cancer Res. 2009; 15:5267-73.

19. Rosell R, Moran T, Queralt C, Porta R, Cardenal F, Camps C, Majem M, Lopez-Vivanco G, Isla D, Provencio M, Insa A, Massuti B, Gonzalez-Larriba JL. Screening for Epidermal Growth Factor Receptor Mutations in Lung Cancer. New Engl J Med. 2009; 361:958.

20. Liu Y, Ren Z, Wang J, Zhang S. Epidermal growth factor receptor-tyrosine kinase inhibitor therapy is especially beneficial to patients with exon 19 deletion compared with exon 21 L858R mutation in non-small-cell lung cancer: Systematic review and meta analysis. Thorac Cancer. 2016; 7:406-14.

21. Ohashi K, Maruvka YE, Michor F, Pao W. Epidermal Growth Factor Receptor Tyrosine Kinase InhibitorResistant Disease. J Clin Oncol. 2013; 31:1070-80.

22. Zheng Z, Jin X, Lin B, Su H, Chen H, Fei S, Zhao L, Deng X, Xie D, Xie C. Efficacy of Second-line Tyrosine Kinase Inhibitors in the Treatment of Metastatic Advanced Nonsmall-cell Lung Cancer Harboring Exon 19 and 21 EGFR Mutations. J Cancer. 2017; 8:597-605.

23. Liu Y, Ren Z, Wang J, Zhang S. Epidermal growth factor receptor-tyrosine kinase inhibitor therapy is especially beneficial to patients with exon 19 deletion compared with exon 21 L858R mutation in non-small-cell lung cancer: Systematic review and meta analysis. Thorac Cancer. 2016; 7:406-14.

24. Yu HA, Arcila ME, Rekhtman N, Sima CS, Zakowski MF, Pao W, Kris MG, Miller VA, Ladanyi M, Riely GJ. Analysis of tumor specimens at the time of acquired resistance to EGFR-TKI therapy in 155 patients with EGFR-mutant lung cancers. Clin Cancer Res. 2013;19:2240-7.

25. Su KY, Chen HY, Li KC, Kuo ML, Yang JC, Chan WK, Ho BC, Chang GC, Shih JY, Yu SL, Yang PC. Pretreatment epidermal growth factor receptor (EGFR) T790M mutation predicts shorter EGFR tyrosine kinase inhibitor response duration in patients with non-small-cell lung cancer. J Clin Oncol. 2012;30:433-40.

26. Ke EE, Zhou Q, Zhang QY, Su J, Chen ZH, Zhang XC, Xu CR, Yang JJ, Tu HY, Yan HH, Zhang YC, Niu FY, Wu YL. A Higher Proportion of the EGFR T790M Mutation May Contribute to the Better Survival of Patients with Exon 19 Deletions Compared with Those with L858R. J Thorac Oncol. 2017; 12:1368-1375.

27. Hu X, Han B, Gu A, Zhang Y, Jiao SC, Wang CL, He J, Jia X, Zhang L, Peng J, Wu M, Ying K, Wang J, et al. A single-arm, multicenter, safety-monitoring, phase IV study of icotinib in treating advanced non-small cell lung cancer (NSCLC). Lung Cancer. 2014; 86:207-12. 